\title{
Long-Term Hematotoxicity After Peptide Receptor Radionuclide Therapy with ${ }^{177}$ Lu-Octreotate
}

\author{
Amir Sabet ${ }^{1}$, Khaled Ezziddin ${ }^{1}$, Ulrich-Frank Pape ${ }^{2}$, Hojjat Ahmadzadehfar ${ }^{1}$, Karin Mayer $^{3}$, Thorsten Pöppel ${ }^{4}$, \\ Stefan Guhlke ${ }^{1}$, Hans-Jürgen Biersack ${ }^{1}$, and Samer Ezziddin ${ }^{1}$ \\ ${ }^{I}$ Department of Nuclear Medicine, University Hospital Bonn, Bonn, Germany; ${ }^{2}$ Department of Hepatology and Gastroenterology, \\ Charité, Campus Virchow Clinic, University Medicine Berlin, Berlin, Germany; ${ }^{3}$ Department of Medicine, University Hospital Bonn, \\ Bonn, Germany; and ${ }^{4}$ Department of Nuclear Medicine, University Hospital Essen, Essen, Germany
}

Myelosuppression may be the dose-limiting toxicity in peptide receptor radionuclide therapy (PRRT). The aim of this study was to investigate the incidence, severity, and reversibility of long-term hematotoxicity in a large cohort of patient undergoing PRRT with ${ }^{177} \mathrm{Lu}$-octreotate for metastatic neuroendocrine tumors. The impact of potential risk factors, including initial cytopenia, advanced bone metastatic disease, previous chemotherapy, and cumulative administered activity, and the protective effects of splenectomy were of particular interest. Methods: A total of 632 PRRT courses were performed in 203 patients with metastatic neuroendocrine tumors. A mean activity of $7.9 \mathrm{GBq}$ of ${ }^{177} \mathrm{Lu}$-octreotate was administered per treatment cycle, with a goal of 4 courses at standard intervals of $3 \mathrm{mo}$. Hematologic parameters were determined before each treatment course, at 2- to 4-wk intervals between the courses, 8-12 wk after the last course of PRRT, and at 3-month intervals for further follow-up. Toxicity was recorded with Common Terminology Criteria for Adverse Events (version 3.0). Results: Myelodysplastic syndrome as a delayed adverse event was documented in 3 patients (1.4\%). Relevant but reversible hematotoxicity (grade 3 or 4 ) occurred in 23 patients $(11.3 \%)$ and 29 administrations (4.6\%), with leukopenia in $2.7 \%$ and thrombocytopenia in $1.7 \%$. The mean time to blood count recovery was 12 mo after the termination of PRRT (range, 3-22 mo). The only preexisting factor that contributed to hematotoxicity was initial cytopenia $(P<0.001)$. A high level of cumulative administered activity $(>29.6 \mathrm{GBq})$ was associated with relevant leukopenia $(P<0.001)$. None of the patients with a history of splenectomy developed grade 3 or 4 hematotoxicity, and splenectomy was inversely associated with the incidence and degree of leukopenia $(P=0.02)$ and thrombocytopenia $(P=0.03)$ Conclusion: PRRT-induced myelosuppression is almost invariably reversible and rarely requires clinical measures. Administered activity and initial cytopenia are the only factors contributing to myelosuppression, whereas splenectomy may exert a protective effect.

Key Words: hematotoxicity; PRRT; ${ }^{177} \mathrm{Lu}$-octreotate; NETs

J Nucl Med 2013; 54:1857-1861

DOI: 10.2967/jnumed.112.119347

Received Dec. 28, 2012; revision accepted May 9, 2013.

For correspondence or reprints contact: Samer Ezziddin, Department of Nuclear Medicine, University Hospital, Sigmund-Freud-Strasse 25, D-53105 Bonn, Germany.

E-mail: samer.ezziddin@ukb.uni-bonn.de

Published online Sep. 5, 2013.

COPYRIGHT (C 2013 by the Society of Nuclear Medicine and Molecular Imaging, Inc.
$\mathbf{P}$ eptide receptor radionuclide therapy (PRRT) with radiolabeled somatostatin analogs is a highly effective systemic treatment in patients with metastatic gastroenteropancreatic neuroendocrine tumors (NETs) (1-9). Bone marrow suppression is regarded as one of the most serious side effects after PRRT (10-13). Because of the lower energy and the shorter tissue penetration of the emitted $\beta$ particles, treatment with ${ }^{177} \mathrm{Lu}$-labeled peptides may result in a lower level of overall toxicity than PRRT with ${ }^{90}$ Y-labeled peptides (14). However, there are only limited data regarding hematotoxicity after PRRT with ${ }^{177} \mathrm{Lu}$-octreotate and contributing risk factors $(2,15,16)$.

The aim of this retrospective study was to investigate the incidence, severity, and reversibility of myelosuppression in a large cohort of patients with metastatic gastroenteropancreatic NETs treated with ${ }^{177} \mathrm{Lu}$-octreotate. The impact of potential risk factors, including the presence of advanced bone metastatic disease, previous chemotherapy, and cumulative administered activity, was of particular interest. The protective impact of splenectomy on the development of hematotoxicity was also explored.

\section{MATERIALS AND METHODS}

A total of 203 consecutive patients treated with ${ }^{177} \mathrm{Lu}$-octreotate at the Department of Nuclear Medicine in Bonn (93 men and 110 women; age range, 34-89 y; mean age, $63 \mathrm{y}$ ) were included in the retrospective analysis. PRRT has been part of standard care in Germany for several years (17). Inclusion criteria for treatment with PRRT were histologically confirmed, unresectable, metastatic NET; sufficient tumor uptake, that is, uptake greater than or equal to that of the liver on baseline receptor imaging; a glomerular filtration rate of greater than $30 \mathrm{~mL} / \mathrm{min} /$ $1.73 \mathrm{~m}^{2}$; a white blood cell count of greater than or equal to $2,000 / \mathrm{mm}^{3}$; a hemoglobin level of greater than or equal to $8 \mathrm{~g} / \mathrm{dL}$; and a platelet level of greater than or equal to $75,000 / \mathrm{mm}^{3}$. A mild reduction in blood counts at baseline was not an exclusion criterion. Seventy-two patients had pancreatic NETs, and 131 patients had nonpancreatic gastroenteropancreatic NETs. All patients showed tumor progression before treatment with ${ }^{177} \mathrm{Lu}$-octreotate. The study was performed in accordance with the Declaration of Helsinki and with national regulations. The local committee on ethics approved this retrospective study, and all patients signed written informed consent.

PRRT

PRRT was performed with a mean of $7.9 \mathrm{GBq}(216 \mathrm{mCi})$ of ${ }^{177} \mathrm{Lu}-$ octreotate per treatment cycle, with the goal of 4 courses at standard intervals of 3 mo (10-14 wk). ${ }^{177} \mathrm{Lu}$ (IDB Holland) had a specific activity in the approximate range of $100-160 \mathrm{GBq} / \mu \mathrm{mol}$ at the time of administration. Peptide labeling $(18,19)$ was performed such that an 
TABLE 1

Prevalence of Examined Factors and Percentage of Patients Developing Significant Toxicity

\begin{tabular}{|c|c|c|c|c|}
\hline \multirow[b]{2}{*}{ Variable } & \multirow[b]{2}{*}{ Total patients $(n)$} & \multicolumn{3}{|c|}{ Patients with significant toxicity $(n)$} \\
\hline & & WBC & PLT & HB \\
\hline \multicolumn{5}{|l|}{ Tumor origin } \\
\hline Pancreatic NET & 72 & $5(6.9)$ & $4(5.0)$ & $4(5.0)$ \\
\hline Gastroenteropancreatic NET & 122 & $7(5.7)$ & $5(4.0)$ & $3(2.4)$ \\
\hline Undetermined primary tumor & 19 & $1(5.2)$ & $1(5.2)$ & $0(0.0)$ \\
\hline Hormonal activity & 96 & $8(10.4)$ & $6(6.2)$ & $4(4.2)$ \\
\hline Chromogranin A level of $>600 \mathrm{ng} / \mathrm{mL}$ & 92 & $7(7.6)$ & $4(4.3)$ & $4(4.3)$ \\
\hline Advanced bone metastases* & 21 & $2(9.5)$ & $0(0.0)$ & $2(9.5)$ \\
\hline Previous chemotherapy & 54 & $4(7.4)$ & $4(7.4)$ & $2(3.7)$ \\
\hline GFR of $<60 \mathrm{~mL} / \mathrm{min} / 1.73 \mathrm{~m}^{2}$ & 35 & $4(11.4)$ & $3(8.6)$ & $2(5.7)$ \\
\hline Age of $\geq 70 y$ & 56 & $2(3.6)$ & $2(3.6)$ & $2(3.6)$ \\
\hline Cumulative dose of $>29.6 \mathrm{GBq}(>800 \mathrm{mCi})$ & 67 & 8 (11.9) & $3(4.5)$ & $4(6.0)$ \\
\hline \multicolumn{5}{|l|}{ Reduction at baseline } \\
\hline WBC & 14 & $4(28.6)$ & & \\
\hline PLT & 37 & & $5(13.5)$ & \\
\hline $\mathrm{HB}$ & 69 & & & $6(8.7)$ \\
\hline Splenectomy & 16 & $0(0.0)$ & $0(0.0)$ & $0(0.0)$ \\
\hline
\end{tabular}

${ }^{\star}$ Advanced bone metastases were defined as multiple uncountable bone lesions.

$\mathrm{WBC}=$ white blood cells; PLT = platelets; $\mathrm{HB}=$ hemoglobin; GFR = glomerular filtration rate.

Data in parentheses are percentages.

apparent specific activity of about $54 \mathrm{GBq} / \mu \mathrm{mol}$ (ratio of activity to the total amount of peptide) was obtained. Nephroprotection was implemented with standard amino acid coinfusion according to the Rotterdam protocol $(15,20)(2.5 \%$ lysine and $2.5 \%$ arginine in $1 \mathrm{~L}$ of $0.9 \%$ $\mathrm{NaCl}$; rate of infusion, $250 \mathrm{~mL} / \mathrm{h}$ ). Hematologic parameters were determined before each treatment course, at 2- to 4-wk intervals between the courses, 8-12 wk after the last course of PRRT, and at 3-month intervals for further follow-up. Toxicity was recorded with the Common Terminology Criteria for Adverse Events (CTCAE), version 3.0.

\section{Statistical Analysis}

The association of treatment-induced myelosuppression (grades 1-4) with the baseline characteristics of the study population and the cumulative administered activity was examined by applying nonparametric tests for independent samples as well as multiple regression analysis. The potential protective effect of splenectomy on the development and degree of hematotoxicity was investigated both in the whole patient cohort and in the subgroup of patients with pancreatic NETs. The statistical software package SPSS (version 18.0; SPSS Inc.) was used to analyze the data.

\section{RESULTS}

A total of 632 PRRT courses were performed in 203 patients (median, 3 treatment courses per patient; cumulative administered activity, 4.9-37.8 GBq). In 47 patients, treatment was stopped after $1(n=18)$ or $2(n=29)$ cycles because of documented disease progression. The median follow-up period was 31 mo $(95 \%$ confidence interval, 20-71) from the start of PRRT. At the start of treatment, 14 patients had leukopenia (11 grade 1 and 3 grade 2),

TABLE 2

Univariate and Multivariate Analyses for Potential Risk Factors Associated with Hematotoxicity

\begin{tabular}{|c|c|c|c|c|c|c|}
\hline \multirow[b]{2}{*}{ Patient characteristic } & \multicolumn{3}{|c|}{ Univariate analysis $P$ value } & \multicolumn{3}{|c|}{ Multivariate analysis $P$ value } \\
\hline & WBC & PLT & $\mathrm{HB}$ & WBC & PLT & $\mathrm{HB}$ \\
\hline Tumor origin & 0.30 & 0.73 & 0.57 & & & \\
\hline Hormonal activity & 0.50 & 0.85 & 0.64 & & & \\
\hline Chromogranin A level of $>600 \mathrm{ng} / \mathrm{mL}$ & 0.53 & 0.321 & 0.16 & & & \\
\hline Advanced bone metastases & 0.84 & 0.22 & 0.25 & & & \\
\hline Previous chemotherapy & 0.49 & 0.20 & 0.98 & & & \\
\hline GFR of $<60 \mathrm{~mL} / \mathrm{min} / 1.73 \mathrm{~m}^{2}$ & 0.09 & 0.11 & 0.06 & & & \\
\hline Age of $\geq 70 y$ & 0.85 & 0.54 & 0.69 & & & \\
\hline Cumulative dose of $>29.6 \mathrm{GBq}(>800 \mathrm{mCi})$ & 0.01 & 0.93 & 0.10 & $<0.001$ & & \\
\hline Initial cytopenia & $<0.001$ & $<0.001$ & $<0.001$ & $<0.001$ & $<0.001$ & $<0.001$ \\
\hline Splenectomy & 0.02 & 0.01 & 0.30 & 0.02 & 0.03 & \\
\hline
\end{tabular}




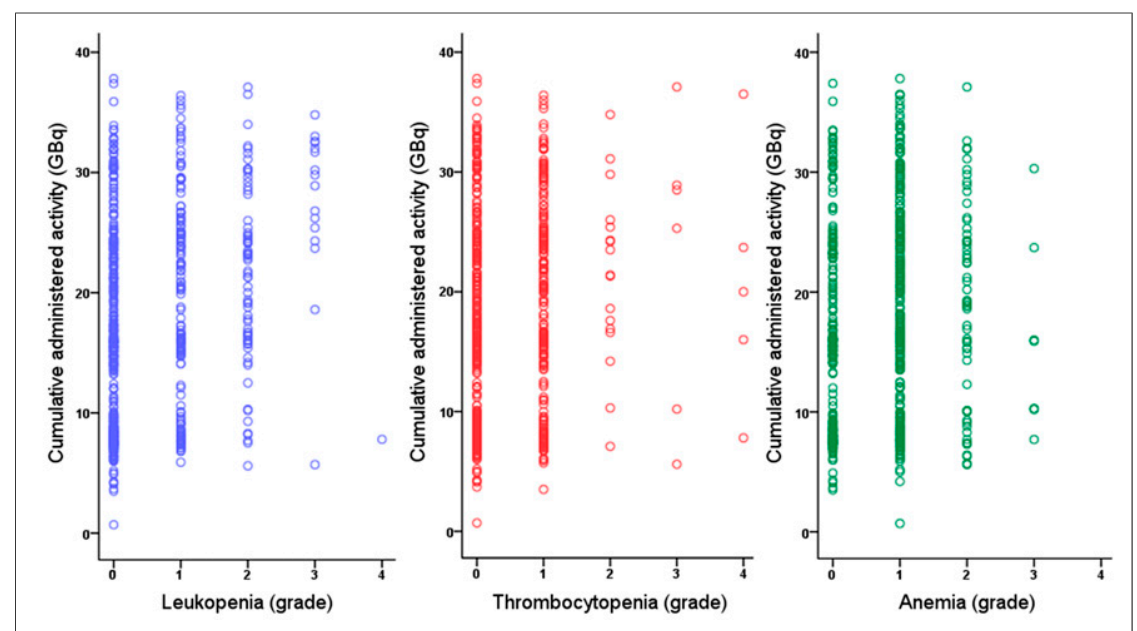

FIGURE 1. Association of cumulative administered activity and incidence of hematotoxicity after each treatment cycle $(n=632)$. Higher cumulative administered activity was a contributing factor for leukopenia.

37 patients had thrombocytopenia (36 grade 1 and 1 grade 2), and 69 patients had anemia (60 grade 1,8 grade 2, and 1 grade 3 ), according to the CTCAE criteria. One patient received PRRT despite having grade 3 anemia at baseline because of the lack of treatment alternatives and progressing tumor disease.

Relevant hematotoxicity (grade 3 or 4 ) occurred 2-8 wk after 29 administrations $(4.6 \%)$ or, expressed per patient, after at least 1 of the administrations in 23 patients $(11.3 \%)$. Seventeen patients showed a disturbance of only 1 hematologic cell line (leukopenia, 9; thrombocytopenia, 5; anemia, 3), whereas 6 patients showed a significant reduction in 2 or all 3 investigated parameters. The most frequently observed adverse event was leukopenia, occurring after 17 administrations (2.7\%); thrombocytopenia occurred after 11 administrations (1.7\%), and anemia occurred after 7 administrations $(1.1 \%)$ (Table 1$)$.

In 8 patients and after 13 administrations, significant bone marrow impairment spontaneously resolved before the next scheduled treatment course. Two patients required blood transfusion during 1 of their hospital stays, and 2 treatment cycles for 1 patient were postponed for 3-4 wk to allow bone marrow recovery. PRRT was

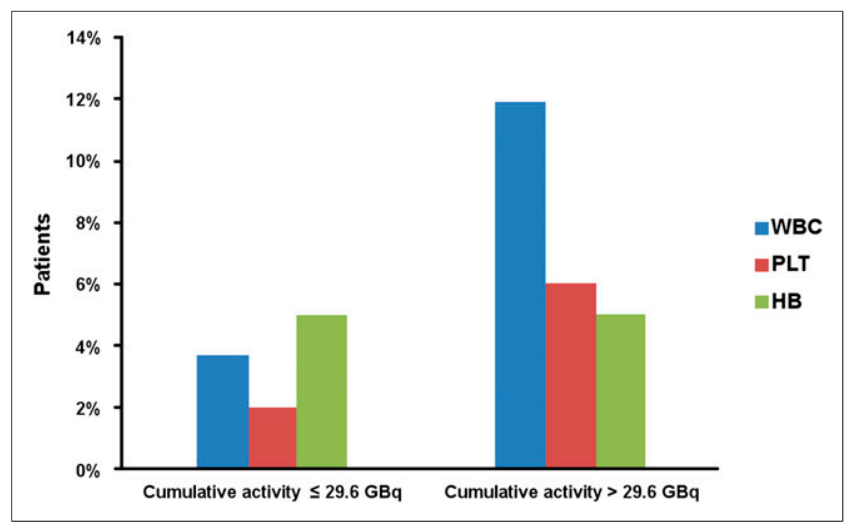

FIGURE 2. Incidence of significant hematotoxicity in patients receiving cumulative administered activities of less than or equal to $29.6 \mathrm{GBq}$ $(\leq 800 \mathrm{mCi})$ and greater than $29.6 \mathrm{GBq}(>800 \mathrm{mCi}) . \mathrm{HB}=$ hemoglobin; $\mathrm{PLT}=$ platelets; $\mathrm{WBC}=$ white blood cells. discontinued in only 1 patient because of aggravated preexisting anemia after the first cycle. This patient had a history of 6 cycles of cisplatin-etoposide until 5 mo before treatment. However, subsequent chemotherapy with 5-fluorouracil-streptozocin was applied 2 mo later and was well tolerated, with no dose-limiting myelosuppression. In 10 patients, relevant myelosuppression was first observed after the completion of the last intended therapy cycle.

Blood values returned to the reference ranges in all but 1 patient, who died 2 mo after the first therapy cycle because of disease progression. The mean time to complete blood count recovery after the termination of PRRT was 12 mo (range, 3-22 mo). Among the baseline characteristics, a pretreatment reduction of hematologic parameters was the only factor associated with an increased rate of hematotoxicity; this observation remained significant after multiple regression analysis (Table 2). A higher level of cumulative activity was a contributing factor for leukopenia, as shown in Figure 1, and patients who received cumulative doses of less than or equal to $29.6 \mathrm{GBq}(800 \mathrm{mCi})$ developed leukopenia less frequently than patients who received doses of greater than 29.6 GBq (800 mCi) $(P<001)$ (Fig. 2). None of the other examined variables, including previous chemotherapy (6-18 mo before PRRT), was associated with the incidence or degree of myelosuppression in our cohort (Table 2).

None of the 16 patients with a history of splenectomy developed hematotoxicity of grade 3 or 4 (Table 3). Statistical analysis showed that splenectomy was inversely associated with the incidence and degree of leukopenia and thrombocytopenia (Fig. 3). Also, in the subgroup of patients with pancreatic NETs $(n=72)$, a history of splenectomy was associated with less frequent hematotoxicity.

Myelodysplastic syndrome as a delayed adverse event, occurring 14, 29, and 34 mo after the termination of PRRT, was documented in 3 patients $(1.4 \%)$. None of the patients had any of the examined risk factors or received a cumulative activity of greater than $29.6 \mathrm{GBq}$. The patients were treated with supportive therapy or chemotherapy, such as lenalidomide. One patient with myelodysplastic syndrome developed acute myeloid leukemia 36 mo after the last treatment cycle; this patient received induction chemotherapy with idarubicin and cytosine arabinoside. None of the patients had died from myelodysplastic syndrome by the time this article was being prepared.

\section{DISCUSSION}

To avoid PRRT-induced bone marrow hypoplasia, a maximum absorbed dose to the bone marrow of 2 Gy has been suggested (20-22). Several models have been applied for bone marrow dosimetry; these include bone marrow aspiration, calculating the dose to the red marrow from the accumulated activity concentration in the blood, and the MIRD scheme. However, all of these methods are associated with specific difficulties and have limited precision. Furthermore, the high interindividual variability in hematologic alterations after the same absorbed dose to the red marrow makes bone marrow dosimetry in clinical practice less valuable 
TABLE 3

PRRT-Induced Toxicities

\begin{tabular}{|c|c|c|c|c|}
\hline \multirow[b]{2}{*}{ Relevant hematotoxicity } & \multicolumn{2}{|c|}{$\begin{array}{l}\text { Incidence } \\
\text { per patient }\end{array}$} & \multicolumn{2}{|c|}{$\begin{array}{l}\text { Incidence } \\
\text { per cycle }\end{array}$} \\
\hline & No. & $\%$ & No. & $\%$ \\
\hline Leukopenia & 13 & 6.4 & 17 & 2.7 \\
\hline Grade 3 & 12 & 5.9 & 16 & 2.5 \\
\hline Grade 4 & 1 & 0.5 & 1 & 0.2 \\
\hline Thrombocytopenia & 10 & 4.9 & 11 & 1.7 \\
\hline Grade 3 & 5 & 2.5 & 6 & 0.9 \\
\hline Grade 4 & 5 & 2.5 & 5 & 0.8 \\
\hline Anemia & 7 & 3.4 & 7 & 1.1 \\
\hline Grade 3 & 7 & 3.4 & 7 & 1.1 \\
\hline Grade 4 & 0 & 0 & 0 & 0 \\
\hline Total & 23 & 11.3 & 29 & 4.6 \\
\hline
\end{tabular}

According to CTCAE.

$(23,24)$. In the present study, relevant hematotoxicity (CTCAE grade 3 or 4 ) occurred after $4.6 \%$ of administrations and in $11.3 \%$ of patients. These results are in agreement with previously published data reporting significant hematotoxicity in $3.6 \%$ of administrations and $9.5 \%$ of 504 patients with metastatic gastroenteropancreatic NETs (2).

The results of this large retrospective analysis indicate the acceptable toxicity profile of PRRT (25-29), which compares favorably with the reported toxicities of common chemotherapy regimens, including 5-fluorouracil-streptozocin (20\%-30\% grade 3 or 4$)$ and sunitinib ( $>30 \%$ grade 3 or 4 ) for pancreatic NETs (30-33). Our observations provide the first evidence for the minor clinical relevance of hematotoxicity after high cumulative administered activities of more than $29.6 \mathrm{GBq}(800 \mathrm{mCi})$ of ${ }^{177} \mathrm{Lu}$-octreotate $(15,16)$. First, myelosuppression was almost invariably reversible (202/203 patients), and although $5 \%$ of patients completed the treatment with significant impairment in at least 1 of the hematologic parameters, complete bone marrow recovery eventually occurred during follow-up. Second, in only 1 individual in the entire cohort did hematotoxicity (in this case, aggravation of preexisting anemia in a patient previously treated with cisplatin-etoposide) lead to discontinuation of treatment.

The only baseline predictor of significant hematotoxicity in the present study was initial cytopenia. In contrast to other studies $(15,34)$, we observed no correlation between a history of previous chemotherapy and the incidence of hematotoxicity (Table 2); this

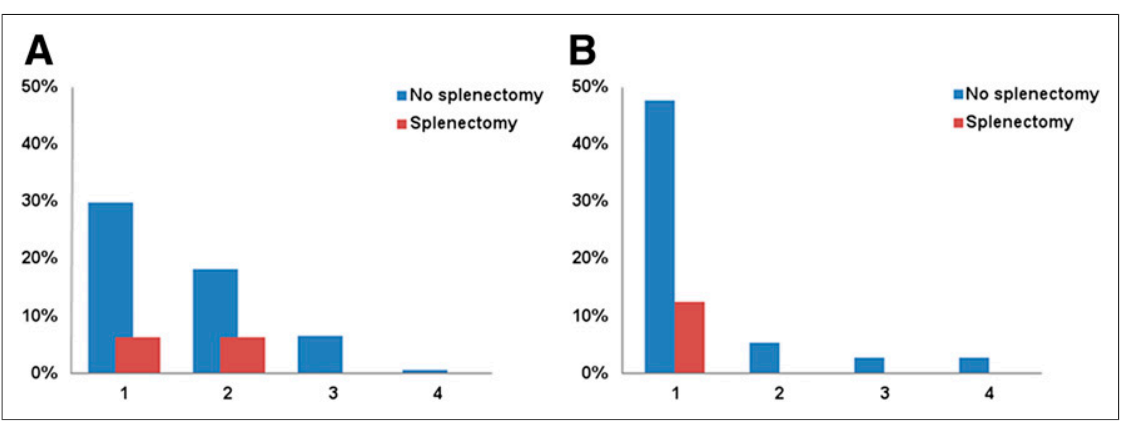

FIGURE 3. Incidence of hematotoxicity in patients with and without history of splenectomy. (A) Grade of leukopenia. (B) Grade of thrombocytopenia. Grades are shown on $x$-axes. finding may encourage the use of PRRT with ${ }^{177}$ Lu-octreotate in groups of patients with generally fewer therapeutic options. Even advanced bone metastases did not promote the risk of hematotoxicity in our cohort $(P>0.2)$. This finding argues against the suggested relative contraindication of advanced bone metastases for PRRT in terms of toxicity and fits well with the excellent response outcome reported earlier for this condition (35).

A high level of cumulative administered activity was associated with increases in the incidence and severity of leukopenia (Fig. 1). This correlation was also observed with lower levels of activity (cumulative doses of $\leq 29.6 \mathrm{GBq}$ [ $\leq 800 \mathrm{mCi}]$ ), in which most treatments will range with currently used protocols $(2,15,16)$. We found no other report in the literature linking the cumulative administered activity of ${ }^{177} \mathrm{Lu}$-octreotate to hematotoxicity. However, as stated before, hematotoxicity was rarely of clinical relevance, even when high administered activities were used.

The spleen is a major reservoir of blood cells and receives the highest absorbed dose of all organs during PRRT, partly because of the presence of somatostatin receptors on lymphocytes $(36,37)$. This intensive radiation may damage blood cells accumulating in the spleen and consequently reduce peripheral blood cell counts. In the present study, splenectomy indeed was shown to have a protective effect against hematotoxicity (Fig. 3). To the best of our knowledge, this is the first study to show the potentially beneficial effect of this condition in patients undergoing PRRT.

In accordance with other studies $(2,20,38)$, myelodysplastic syndrome was observed as a rare delayed side effect in 3 patients $(1.4 \%)$ and at least 14 mo after the termination of PRRT. Two of the 3 patients were still alive at the end of the study (followup: 32-56 mo). Considering the limited prognosis of patients with advanced metastatic NETs, the low risk of this late side effect should not represent a major argument against performing PRRT.

Selective targeting of B cells has been proposed to be the main cause of lymphopenia after PRRT (39). Unfortunately, differential blood counts were not available for our patients; therefore, alterations in this subset of leukocytes could not be analyzed. However, the main limitation of the present study was the retrospective setting. Nevertheless, because of the general lack of prospective studies in the field of PRRT, retrospective analyses of large cohorts of patients may be regarded as the best available alternative.

\section{CONCLUSION}

The overall incidence of PRRT-induced myelosuppression was acceptable, and this condition had a transient nature, even after the use of high levels of cumulative administered activities ( $>29.6$ $\mathrm{GBq})$. Besides administered activity, initial cytopenia was the only factor contributing to myelosuppression, whereas splenectomy may exert a protective effect. Contrary to the findings of previous studies, we found that advanced osseous metastatic disease and previous chemotherapy did not increase the rate of hematotoxicity and should not preclude patients from receiving PRRT with ${ }^{177} \mathrm{Lu}$-octreotate.

\section{DISCLOSURE}

The costs of publication of this article were defrayed in part by the payment of 
page charges. Therefore, and solely to indicate this fact, this article is hereby marked "advertisement" in accordance with 18 USC section 1734. No other potential conflict of interest relevant to this article was reported.

\section{ACKNOWLEDGMENTS}

We are grateful to Professor Eric Krenning, Professor Dik Kwekkeboom, and Professor Wouter A.P. Breeman (Erasmus Medical Center, Rotterdam, The Netherlands) for sharing their experience in the receptor targeting field and making somatostatin receptormediated treatment at our institution possible. We also thank Professor Richard P. Baum (Department of Nuclear Medicine and PET Center, Zentralklinik, Bad Berka, Germany) for his critical and constructive input in this field. We also are grateful to the personnel of the Department of Nuclear Medicine in Bonn and especially to the nursing staff of the therapy ward.

\section{REFERENCES}

1. Kwekkeboom DJ, de Herder WW, van Eijck CH, et al. Peptide receptor radionuclide therapy in patients with gastroenteropancreatic neuroendocrine tumors. Semin Nucl Med. 2010;40:78-88.

2. Kwekkeboom DJ, de Herder WW, Kam BL, et al. Treatment with the radio-

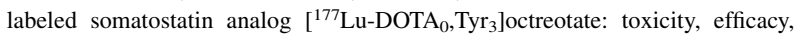
and survival. J Clin Oncol. 2008;26:2124-2130.

3. Kwekkeboom DJ, de Herder WW, Krenning EP. Somatostatin receptor-targeted radionuclide therapy in patients with gastroenteropancreatic neuroendocrine tumors. Endocrinol Metab Clin North Am. 2011;40:173-185.

4. Buscombe JR, Caplin ME, Hilson AJ. Long-term efficacy of high-activity ${ }^{111} \mathrm{In}$ pentetreotide therapy in patients with disseminated neuroendocrine tumors. $\mathrm{J}$ Nucl Med. 2003;44:1-6.

5. Delpassand ES, Samarghandi A, Mourtada JS, et al. Long-term survival, toxicity profile, and role of F-18 FDG PET/CT scan in patients with progressive neuroendocrine tumors following peptide receptor radionuclide therapy with high activity In-111 pentetreotide. Theranostics. 2012;2:472-480.

6. Waldherr C, Pless M, Maecke HR, Haldemann A, Mueller-Brand J. The clinical value of [ ${ }^{90}$ Y-DOTA]-D-Phe1-Tyr3-octreotide ( ${ }^{90}$ Y-DOTATOC) in the treatment of neuroendocrine tumours: a clinical phase II study. Ann Oncol. 2001;12:941-945.

7. Waldherr C, Pless M, Maecke HR, et al. Tumor response and clinical benefit in neuroendocrine tumors after 7.4 GBq ${ }^{90}$ Y-DOTATOC. J Nucl Med. 2002;43:610-616.

8. Iten F, Müller B, Schindler C, et al. Response to [ ${ }^{90}$ yttrium-DOTA]-TOC treatment is associated with long-term survival benefit in metastasized medullary thyroid cancer: a phase II clinical trial. Clin Cancer Res. 2007;13:6696-6702.

9. Ezziddin S, Opitz M, Attassi M, et al. Impact of the Ki-67 proliferation index on response to peptide receptor radionuclide therapy. Eur J Nucl Med Mol Imaging. 2011;38:459-466.

10. Paganelli G, Bodei L, Handkiewicz Junak D, et al. ${ }^{90}$ Y-DOTA-D-Phe1-Try3-octreotide in therapy of neuroendocrine malignancies. Biopolymers. 2002;66:393-398.

11. Otte A, Herrmann R, Heppeler A, et al. Yttrium-90 DOTATOC: first clinical results. Eur J Nucl Med. 1999;26:1439-1447.

12. Bodei L, Cremonesi M, Grana C, et al. Receptor radionuclide therapy with ${ }^{90} \mathrm{Y}-$ [DOTA $]_{0}-\mathrm{Tyr}_{3}$-octreotide $\left({ }^{90} \mathrm{Y}\right.$-DOTATOC) in neuroendocrine tumours. Eur $J$ Nucl Med Mol Imaging. 2004;31:1038-1046.

13. Imhof A, Brunner P, Marincek N, et al. Response, survival, and long-term toxicity after therapy with the radiolabeled somatostatin analogue $\left[{ }^{90} \mathrm{Y}-\mathrm{DOTA}\right]-$ TOC in metastasized neuroendocrine cancers. J Clin Oncol. 2011;29:2416-2423.

14. Kam BL, Teunissen JJ, Krenning EP, et al. Lutetium-labelled peptides for therapy of neuroendocrine tumours. Eur J Nucl Med Mol Imaging. 2012;39(suppl 1): S103-S112.

15. Kwekkeboom DJ, Teunissen JJ, Bakker WH, et al. Radiolabeled somatostatin analog $\left[{ }^{177} \mathrm{Lu}-\mathrm{DOTA}_{0}, \mathrm{Tyr}_{3}\right]$ octreotate in patients with endocrine gastroenteropancreatic tumors. J Clin Oncol. 2005;23:2754-2762.

16. Bodei L, Cremonesi M, Grana CM, et al. Peptide receptor radionuclide therapy with ${ }^{177}$ Lu-DOTATATE: the IEO phase I-II study. Eur J Nucl Med Mol Imaging. 2011;38:2125-2135.
17. Hörsch D, Ezziddin S, Haug A, et al. Peptide receptor radionuclide therapy for neuroendocrine tumors in Germany: first results of a multi-institutional cancer registry. Recent Results Cancer Res. 2013;194:457-465.

18. Breeman WA, De Jong M, Visser TJ, Erion JL, Krenning EP. Optimising conditions for radiolabelling of DOTA-peptides with ${ }^{90} \mathrm{Y},{ }^{111} \mathrm{In}$ and ${ }^{177} \mathrm{Lu}$ at high specific activities. Eur J Nucl Med Mol Imaging. 2003;30:917-920.

19. Breeman WA, van der Wansem K, Bernard BF, et al. The addition of DTPA to $\left[{ }^{177} \mathrm{Lu}-\mathrm{DOTA} \mathrm{A}_{0}, \mathrm{Tyr}_{3}\right]$ octreotate prior to administration reduces rat skeleton uptake of radioactivity. Eur J Nucl Med Mol Imaging. 2003;30:312-315.

20. Kwekkeboom DJ, Bakker WH, Kam BL, et al. Treatment of patients with gastroentero-pancreatic (GEP) tumours with the novel radiolabelled somatostatin analogue $\left[{ }^{177} \mathrm{Lu}_{-\mathrm{DOTA}_{0}}, \mathrm{Tyr}_{3}\right]$ octreotate. Eur J Nucl Med Mol Imaging. 2003; 30:417-422.

21. Wessels BW, Bolch WE, Bouchet LG, et al. Bone marrow dosimetry using blood-based models for radiolabeled antibody therapy: a multiinstitutional comparison. J Nucl Med. 2004;45:1725-1733.

22. Cremonesi M, Ferrari M, Zoboli S, et al. Biokinetics and dosimetry in patients administered with ${ }^{111} \mathrm{In}$-DOTA-Tyr ${ }_{3}$-octreotide: implications for internal radiotherapy with ${ }^{90}$ Y-DOTATOC. Eur J Nucl Med. 1999;26:877-886.

23. Forrer F, Krenning EP, Kooij PP, et al. Bone marrow dosimetry in peptide receptor radionuclide therapy with $\left[{ }^{177} \mathrm{Lu}_{-\mathrm{DOTA}_{0}}, \mathrm{Tyr}_{3}\right]$ octreotate. Eur J Nucl Med Mol Imaging. 2009;36:1138-1146.

24. Kwekkeboom DJ, Bakker WH, Kooij PP, et al. $\left[{ }^{177}\right.$ Lu-DOTAOTyr $\left._{3}\right]$ octreotate: comparison with $\left[{ }^{111} \mathrm{In}_{-} \mathrm{DTPA}_{0}\right]$ octreotide in patients. Eur J Nucl Med. 2001; 28:1319-1325.

25. Chinol M, Bodei L, Cremonesi M, Paganelli G. Receptor-mediated radiotherapy with Y-DOTA-DPhe-Tyr-octreotide: the experience of the European Institute of Oncology Group. Semin Nucl Med. 2002;32:141-147.

26. Bodei L, Cremonesi M, Zoboli S, et al. Receptor-mediated radionuclide therapy with ${ }^{90}$ Y-DOTATOC in association with amino acid infusion: a phase I study. Eur J Nucl Med Mol Imaging. 2003;30:207-216.

27. Anthony LB, Woltering EA, Espenan GD, Cronin MD, Maloney TJ, McCarthy KE. Indium-111-pentetreotide prolongs survival in gastroenteropancreatic malignancies. Semin Nucl Med. 2002;32:123-132.

28. Valkema R, Pauwels S, Kvols LK, et al. Survival and response after peptide receptor radionuclide therapy with $\left[{ }^{90} \mathrm{Y}-\mathrm{DOTA}_{0}, \mathrm{Tyr}_{3}\right]$ octreotide in patients with advanced gastroenteropancreatic neuroendocrine tumors. Semin Nucl Med. 2006; 36:147-156.

29. Villard L, Romer A, Marincek N, et al. Cohort study of somatostatin-based radiopeptide therapy with $\left[{ }^{90} \mathrm{Y}-\mathrm{DOTA}\right]-\mathrm{TOC}$ versus $\left[{ }^{90} \mathrm{Y}-\mathrm{DOTA}\right]-\mathrm{TOC}$ plus $\left[{ }^{177} \mathrm{Lu}-\right.$ DOTA]-TOC in neuroendocrine cancers. J Clin Oncol. 2012;30:1100-1106.

30. Engstrom PF, Lavin PT, Moertel CG, Folsch E, Douglass HO Jr. Streptozocin plus fluorouracil versus doxorubicin therapy for metastatic carcinoid tumor. $J$ Clin Oncol. 1984;2:1255-1259.

31. Turner NC, Strauss SJ, Sarker D, et al. Chemotherapy with 5-fluorouracil, cisplatin and streptozocin for neuroendocrine tumours. Br J Cancer. 2010;102: 1106-1112.

32. Kulke MH, Lenz HJ, Meropol NJ, et al. Activity of sunitinib in patients with advanced neuroendocrine tumors. J Clin Oncol. 2008;26:3403-3410.

33. Moertel CG, Lefkopoulo M, Lipsitz S, Hahn RG, Klaassen D. Streptozocindoxorubicin, streptozocin-fluorouracil or chlorozotocin in the treatment of advanced islet-cell carcinoma. $N$ Engl J Med. 1992;326:519-523.

34. Kwekkeboom DJ, Kam BL, van Essen M, et al. Somatostatin-receptor-based imaging and therapy of gastroenteropancreatic neuroendocrine tumors. Endocr Relat Cancer. 2010;17:R53-R73.

35. Ezziddin S, Sabet A, Heinemann F, et al. Response and long-term control of bone metastases after peptide receptor radionuclide therapy with ${ }^{177} \mathrm{Lu}$-octreotate. J Nucl Med. 2011;52:1197-1203.

36. Reubi JC, Kvols L, Krenning E, Lamberts SW. Distribution of somatostatin receptors in normal and tumor tissue. Metabolism. 1990;39:78-81.

37. Wehrmann C, Senftleben S, Zachert C, Muller D, Baum RP. Results of individual patient dosimetry in peptide receptor radionuclide therapy with ${ }^{177} \mathrm{Lu}$ DOTATATE and ${ }^{177}$ Lu DOTA-NOC. Cancer Biother Radiopharm. 2007;22:406-416.

38. Valkema R, De Jong M, Bakker WH, et al. Phase I study of peptide receptor radionuclide therapy with [In-DTPA] octreotide: the Rotterdam experience. Semin Nucl Med. 2002;32:110-122.

39. Sierra ML, Agazzi A, Bodei L, et al. Lymphocytic toxicity in patients after peptide-receptor radionuclide therapy (PRRT) with ${ }^{177} \mathrm{Lu}$-DOTATATE and ${ }^{90} \mathrm{Y}$ DOTATOC. Cancer Biother Radiopharm. 2009;24:659-665. 